\title{
Head and Neck Cancer Research Ethics: A Primer
}

\author{
1Joshua D Smith, ${ }^{2}$ Kayte Spector-Bagdady, ${ }^{3}$ Andrew G Shuman
}

\begin{abstract}
The rapidly evolving landscape of clinical research in head and neck cancer promises scientific discovery that will benefit patients and stand to improve the human condition for years to come. However, it is imperative that the head and neck oncology research community remains cognizant of the ethical conundrums posed by novel clinical investigation and respects and honors the primacy of the patients and participants upon whom such discoveries depend. It is as vital as ever to revisit the ethical debates of the past, the novel issues of the present, and potential ethical concerns of the future, to ensure research subject protection and respect evolves at the same pace as the research enterprise itself. Herein, we review the basic ethical principles required of human subjects research, the regulatory landscape, and selected emerging debates with relevant examples for head and neck providers and researchers.
\end{abstract}

Keywords: Clinical research, Head \& neck cancer, IRBs, Research eithics.

How to cite this article: Smith JD, Spector-Bagdady K, Shuman AG. Head and Neck Cancer Research Ethics: A Primer. Int J Head Neck Surg 2017;8(2):49-56.

Source of support: Nil

Conflict of interest: None

\section{INTRODUCTION}

There has been a veritable explosion in the volume and breadth of clinical research conducted with human subjects across oncologic disciplines. Moreover, scientific and technological advances coupled with globalization

\footnotetext{
${ }^{1}$ Student, ${ }^{2,3}$ Assistant Professor

${ }^{1}$ Head and Neck Translational Oncology Laboratory Comprehensive Cancer Center, University of Michigan Medical School; Department of Otolaryngology-Head and Neck Surgery University of Michigan Medical School, Ann Arbor, Michigan

${ }^{2}$ Center for Bioethics and Social Sciences in Medicine, University of Michigan Medical School; Program in Sexual Rights and Reproductive Justice in the Department of Obstetrics and Gynecology, University of Michigan Medical School, Ann Arbor Michigan

${ }^{3}$ Head and Neck Translational Oncology Laboratory Comprehensive Cancer Center, University of Michigan Medical School; Department of Otolaryngology-Head and Neck Surgery University of Michigan Medical School; Center for Bioethics and Social Sciences in Medicine, University of Michigan Medical School; Program in Sexual Rights and Reproductive Justice in the Department of Obstetrics and Gynecology, University of Michigan Medical School, Ann Arbor, Michigan
}

Corresponding Author: Andrew G Shuman, 1904 Taubman Center; 1500 E Medical Center Drive, Ann Arbor, Michigan 48109, Phone: (734)232-0120, e-mail: shuman@med.umich.edu have fundamentally altered the scope and nature of cancer research worldwide. For example, randomized controlled trials, the gold standard of clinical research, are trending toward a collaborative model in which patient recruitment and study monitoring occur across multiple academic institutions as a means to reduce logistical and financial challenges of implementation and generate robust, generalizable study results. ${ }^{1}$ Further, globalization of clinical trials, a somewhat recent phenomenon, continues at a staggering pace as academic institutions and pharmaceutical companies attempt to minimize overhead costs, accelerate patient recruitment in populationdense countries, and mitigate regulatory barriers. ${ }^{2}$ In biospecimen (e.g., excised tumors) research, cutting-edge techniques, such as cell line research, whole-genome sequencing, and the clustered regularly interspaced short palindromic repeats (CRISPR)-Cas9 gene-editing system, are revolutionizing the depth and breadth of scientific data generated from large biorepositories. ${ }^{3}$ Electronic health records and "big data" are now recognized as a rich, immediate, and low-risk source of information and are increasingly employed in various and sophisticated clinical study designs. ${ }^{4,5}$

This rapid growth and diversification similarly applies to head and neck cancer research. Recent trials have demonstrated improved survival in patients with recurrent squamous cell carcinoma of the head and neck after treatment with the programmed death 1 inhibitor nivolumab vs standard platinum-based chemotherapy. ${ }^{6}$ Similarly, high-quality evidence demonstrates that selected patients with advanced stage squamous cell carcinoma treated nonsurgically can avoid posttreatment neck dissection. ${ }^{7}$ The survival benefit of elective neck dissection in earlystage oral cavity cancer has similarly been established. ${ }^{8}$ Additionally, biological therapies are translating everfaster from scientific hypotheses to clinical utility, illustrating the rapidity and ease of the bench-to-bedside-and-back model in head and neck cancer. ${ }^{9-11}$ Indeed, the traditional labels of "clinician," "scientist," "patient," and "research subject" are becoming blurred and intertwined.

Studies, such as these demonstrate the irrefutable value of scientifically valid, ethically conducted clinical trials.

Our patients remain at the center of this paradigm shift in head and neck cancer research. Indeed, the morphing landscape of head and neck cancer research forecasts the discovery of new genetic aberrations and pathophysiologic mechanisms of malignancy, the development of 
novel oncologic treatments tailored to individual patients, and improvement in quality of life and overall survival. However, these advancements must be met with a responsive, adaptive, and equally critical discourse on the ethical implications of a changing research landscape. It is as vital as ever to revisit the ethical debates of the past, the novel issues of the present, and potential ethical concerns of the future, to ensure research subject protection and respect evolves at the same pace as the research enterprise itself.

The scope of human subjects research ethics is massive, and no single document can encompass or summarize all necessary components. However, stakeholders require a working understanding of how broader research ethics principles apply to individual investigators and studies, as well as which current issues are particularly relevant and vexing. Herein, we review the basic ethical principles required of human subjects research, the regulatory landscape, and selected emerging debates with relevant examples for head and neck providers and researchers.

\section{CLINICAL RESEARCH ETHICS}

It is difficult to concisely summarize the main tenets of what makes clinical research ethical (or conversely, what would constitute an unacceptable breach of ethical conduct in research). In general, the research ethics community considers human subjects research quite differently from research involving animals or nonliving materials. We will focus our discussion on human subjects research, recognizing that head and neck cancer investigators will certainly encounter many other types of research platforms; each with distinct rules, regulations, and ethical analysis.

First, we must approach an overview of clinical research ethics with recognition that when patients or volunteers become "research subjects," an important shift in focus occurs. Whereas the foremost goal of clinical care is the best interests of the patient in front of us, the foremost goal of clinical research is to test a hypothesis in order to benefit future patient populations. Thus, there can be conflicting obligations: The need to protect the well-being of the research subject and to address the aims of the study itself. As such, the primary goal of clinical medicine and clinical research is fundamentally different; acknowledgment of this fact is critical to a constructive discourse on clinical research ethics. This becomes particularly difficult when a clinician is also a member of the research team, in which case the provider/ investigator patient relationship may unduly influence ethical and compassionate delivery of care.

Despite the variety inherent in purpose, design, execution, and interpretation of clinical research, there are dominant constructs that form the foundation for ethical research with human subjects. In a landmark review, Emanuel et al ${ }^{12}$ outlined seven "ethical requirements" to summarize governmental and independent regulatory guidance on ethical conduct of research and attempt to synthesize such guidance into a coherent, practical précis. They are as follows:

- Value: To be ethical, clinical research must have the potential to lead to improvements in health or well-being.

- Scientific validity: Clinical research conducted with poor, unreproducible experimental design is invalid, and therefore, unethical.

- Fair subject selection: The scientific goals of the study must drive participant selection, rather than vulnerability or privilege of certain socioeconomic or cultural groups.

- Favorablerisk-benefitratio: Benefits must be enhanced, risks must be minimized, and benefits must outweigh risks.

- Independent review: Individuals with no affiliation to research study must appraise and amend the study to meet ethical requirements.

- Informed consent. Study participants must control whether or not to participate in clinical research; must be accurately informed of the risks, benefits, and purpose of research; and be allowed to rescind their participation at any point.

- Respect for enrolled subjects: Participant privacy should be respected throughout the study duration and their well-being closely monitored. ${ }^{12}$

As summarized, these requirements are the backbone for the ethical conduct of clinical research involving human subjects. Clinical research that neglects or omits one or more of these requirements raises considerable concern. While these categories reflect core values inherent to many cultures, they must be interpreted and implemented in the context of societal and cultural differences as well as the specific nature of the investigations being proposed.

\section{THE REGULATORY LANDSCAPE}

The history of human subjects research is tainted by infamous and tragic trespasses against human autonomy, frank lapses in moral and ethical conduct on behalf of clinicians and researchers, and exploitation of vulnerable patient populations. ${ }^{13,14}$ In response, several regulatory codes have emerged over the past half-century. The Nuremberg Code and the Declaration of Helsinki were each drafted to provide a guiding set of moral and ethical principles to guide researchers in designing and conducting studies using human subjects. ${ }^{15,16}$ The most recent iteration of the Declaration of Helsinki, published in 2013, maintained the core principles of the original document and was targeted to a global audience. ${ }^{16}$ As 
such, it reaffirms that researchers are ethically bound to respect patient autonomy, health, dignity, integrity, privacy, and right to self-determination and to facilitate a noncoercive, informative, and voluntary informed consent procedure for clinical research. Owing to the increasingly collaborative nature of the clinical research enterprise, new stipulations were added emphasizing the scientific community's responsibility to publicly disseminate positive, negative, and inconclusive results for critical appraisal and for the general benefit of society.

Similarly targeted to a multinational audience of clinical researchers is the "International Ethical Guidelines for Health-Related Research Involving Human Subjects," published by the Council for International Organizations of Medical Sciences in association with the World Health Organization. ${ }^{17}$ Newly updated in 2016 to reflect heightened influence on translational research paradigms and increases in "big data" research, this document seeks to address how to "apply universal ethical principles to biomedical research in a multicultural world with a multiplicity of health care systems." Ethical standards in different areas, such as choice of control in clinical trials; patient confidentiality; collection, storage, and use of biological materials and patient health data; and research with special populations (e.g., children, those unable to provide informed consent) are set forth.

The 1979 Belmont Report provided the ethical foundation for clinical research in the United States. The Belmont Report delineated how best to uphold the three timeless principles of respect for persons, beneficence, and justice in the areas of informed consent, risks and benefits of study participation, and selection of study participants. ${ }^{18}$ The Federal Policy for the Protection of Human Research Subjects, subpart A of which is referred to as the "Common Rule" because of its adoption across many federal departments and agencies, reflects the Belmont Report's themes and mandates methods of implementation for federally funded human subjects research.

The purpose of the Common Rule is to "protect human research subjects while facilitating valuable research and reducing burden, delay, and ambiguity for investigators."19 It does so by providing an overarching regulatory framework for informed consent procedures and Institutional Review Board (IRB) function, and operates and mandates compliance by federally funded researchers. Following several years of thoughtful and passionate commentary from the US research patient/participant community, an updated Common Rule was recently published by the Department of Health and Human Services. ${ }^{19}$ The recent iteration of the Common Rule specifies new language to be included in informed consent procedures, allows for broad consent for future research of identifiable human data and biospecimens, and establishes new requirements for IRB approval and monitoring of clinical trials occurring at multiple clinical sites. ${ }^{19}$

At the local level, an IRB is typically comprised of individuals of diverse backgrounds and remains the regulatory body tasked with the responsibility of critically analyzing research involving human subjects for its ethical merits and necessary protections. Specific roles of IRBs include protecting and ensuring the rights of human subjects, ensuring that risks of study participation are minimal, guaranteeing subject confidentiality, monitoring for adverse events of clinical trials, and assessing for conflicts of interests of investigators. ${ }^{20}$ The IRBs in the United States are regulated and empowered through the Federal government; internationally, regulatory and institutional policies vary.

\section{CLINICAL TRIALS AND EQUIPOISE}

Prospective clinical trials may take myriad forms and structures. Interventional trials evaluating therapeutic efficacy (e.g., of a new drug or surgical procedure) that involve multiple study arms are typically considered differently than purely observational or single-arm studies. These designs may include a placebo or other comparative control group. Despite their ubiquity, debate persists regarding the ethics of randomization, specific to how different trial arms are constructed and developed. ${ }^{21-23}$

Central to this discussion is the concept of clinical equipoise. Equipoise presumes that the comparative therapeutic merit of different arms of a clinical trial (e.g., Drug X vs Drug Y, or Drug Z vs placebo) is genuinely uncertain. Equipoise obligates that superiority of one treatment over another is unknown to the trial investigator and/or there exists controversy or uncertainty in the broader scientific community at large regarding therapeutic efficacy. ${ }^{24}$ Equipoise, a basic means of ensuring universal, fair participant treatment and minimizing unacceptable or unnecessary risks to research subjects, must be maintained throughout the duration of a clinical trial. At any time, should there arise convincing evidence of treatment superiority in one trial arm vs another, the condition of equipoise no longer exists, and thus the study must cease (thereby generating the concept of "early stopping rules" and data safety monitoring). ${ }^{24}$

Low-risk papillary thyroid cancer is a contemporary paradigm for the consideration of clinical equipoise in our field. There is a skyrocketing global prevalence of micropapillary thyroid cancer (i.e., $<1 \mathrm{~cm}$ ) attributable to incidentally detected thyroid nodules and aggressive biopsies thereof. ${ }^{25,26}$ The vast majority of such neoplasms remain indolent, and while surgery for micropapillary thyroid cancer results in exceptionally low locoregional recurrence $(<5 \%)$, distant recurrence $(1-2 \%)$, and disease-specific mortality $(<1 \%)$, this may be attributable to the indolent nature of the disease 
rather than the effectiveness of treatment. ${ }^{27}$ Nascent data establish the safety of close surveillance and avoiding surgical management in selected cases; two prospective studies showed minimal growth of primary tumors and rare nodal metastases (all of which were detected and salvaged) in a cohort of patients with micropapillary thyroid cancer. ${ }^{28,29}$ Nevertheless, watchful waiting of even indolent cancers is not yet accepted by providers. ${ }^{30}$ Recently, a spirited discussion has blossomed regarding the appropriateness of immediate surgical resection vs close clinical surveillance in this patient population. ${ }^{31}$

This dynamic and fluid conversation among head and neck surgeons offers questions regarding equipoise in proposed clinical trials evaluating observation vs surgery for selected cases of low-risk thyroid cancer. Paramount is how we consider relative safety of such variant clinical pathways, and how the inherent risk of an operation can be compared with the small risk of oncologic progression. While randomized controlled trials may seem obligatory, they are simply infeasible due to necessary statistical power, coupled with patients' strong reticence to consent to be randomized among such disparate arms. In that case, what level of data is sufficient to integrate close clinical surveillance into routine clinical practice? While not a research ethics question per se, prospective observational data may be sufficient not only to achieve equipoise but also to establish an acceptable clinical pathway.

\section{INDUSTRY RELATIONSHIPS, CONFLICTS OF INTEREST, AND DISCLOSURES}

Relationships between clinician-researchers and industry, particularly those companies invested in pharmaceuticals and medical devices, have only gotten more intertwined. In the past decade, the number of industry-sponsored clinical trials has risen significantly concomitant with a substantial decrease in National Institutes of Health-funded, "notfor-profit" studies. ${ }^{32}$ The reasons for this shift are myriad, ranging from decline in governmental appropriation of funds to soaring costs for patient recruitment and trial implementation and monitoring. ${ }^{33}$ Justifiably, many have raised concerns centered around the ethics of industrysponsored clinical research, disclosure, and minimization of conflicts of interest, and validity and generalizability of trial results. Given vast financial investment and need for positive, profitable study results inherent in industrysponsored trials, some have raised ethical concerns related to investigator objectivity and clinical equipoise. ${ }^{34}$

At the helm of any clinical study, industry-sponsored or independent, is the clinician-researcher, who has an ethical and professional responsibility to all stakeholders involved, namely patients/research subjects in addition to their funding source and the academic community. In spite of the inherent conflict of interest in industry-sponsored trials, clinician-researchers must commit themselves to practicing honesty, integrity, and openness in informed consent procedures, patient interactions, data analysis and interpretation, and objective reporting of trial results and implications. ${ }^{35}$ Disclosure, the process of divulging all real and potential conflicts of interest, has long been accepted as a simple and sufficient method of curtailing bias and assuring colleagues of objectivity in peer-reviewed science. However, disclosure (i.e., transparency) does not alone ensure objectivity and appropriateness. ${ }^{36}$ Stakeholders must be particularly cognizant of subtle biases and evidence of undisclosed conflicts of interest (e.g., repeated use of a brand name, overemphasis on critiquing competitor's product) in order to ensure the propagation of ethically sound, valid knowledge in industry-sponsored clinical trials, and debate remains over whether this is ever possible. ${ }^{37}$

The American Academy of Otolaryngology - Head and Neck Surgery (AAO-HNSF), recognizing the importance of ethical disclosure in increasingly utilized clinical practice guidelines, provides detailed instructions for appropriate conflict of interest disclosure and management of such conflicts. ${ }^{38}$ The validity and utility of clinical practice guidelines in otolaryngology derive from the rigorous, detailed, and comprehensive review of the research literature by content experts, patients and patient advocates, and other stakeholders. ${ }^{39}$ As such, clinical practice guidelines represent an objective, evidence-based summary of years of methodologically sound clinical research and continue to gain influence in contemporary patient care in otolaryngology and beyond. ${ }^{39}$ Industry sponsorship of clinical practice guidelines has emerged as a mutually beneficial means of allaying often exorbitant costs of guideline development for organizations (e.g., AAO-HNSF) while providing an investment for industry partners to influence data interpretation and reporting, and ultimately clinical practice. ${ }^{40}$

Though there exists only minimal research on the methods of data collection and quality of final guidelines in industry-sponsored clinical practice guidelines, there is a legitimate concern regarding potential conflicts of interest unduly influencing interpretation and summation of valid, empiric research data. ${ }^{40}$ With a growing influence of such guidelines in clinical practice (there are currently 13 published by the AAO-HNSF), we must remain cognizant of conflicts of interest and appropriate disclosure when industry becomes involved to ensure propagation of valid, ethically sound, and patient-centered guidelines. ${ }^{41}$

\section{ETHICS OF SURGICAL INNOVATION}

The process of introducing and approving a new pharmaceutical intervention for a given medical condition is 
elaborate, expensive, rigorous, and iterative. Undertaken in multiple phases and typically involving multiple clinical trials, development of new pharmaceuticals requires strict monitoring and regulation at multiple levels according to a well-structured paradigm.

However, innovative techniques and technology present unique conceptual problems in surgery. Placebocontrolled trials (e.g., surgery vs sham surgery) or activecontrol trials (e.g., surgery X vs surgery $\mathrm{Y}$ ) are often impossible due to the absence of equipoise, patient and clinician reticence to undergo randomization, existing effective therapies, and frequent lack of statistical power in modestly sized populations. ${ }^{42}$ Additionally, a doubleblinded design is typically impossible, and sham surgery is particularly contentious. ${ }^{43}$

Surgeons routinely practice innovation, demanded to remain adaptive and creative in tailoring procedures to diverse individuals with complexities and nuances obvious to those familiar with the operating room. It follows then that surgical innovation is often spontaneous or serendipitous, initially lacking objective evaluation of its utility, safety, and ethical merits, save for in the surgeon's own mind. But how do we reconcile these realities with research ethical principles? In recent years, there has been an important and justifiable movement in the surgical community toward increased regulation of surgical innovation to ensure patient autonomy and protection. This movement seeks to analyze and solve two fundamental questions: What ethical issues arise from surgical innovation, and how can surgical innovation be regulated in such a way as to guarantee ethical surgical practice while not stifling innovation $?^{44}$

To address the first question, a recent meta-analysis identified four main areas of ethical concern surrounding surgical innovation: Oversight, informed consent, technical learning curves, and innovative procedures in vulnerable patients. ${ }^{45}$ While there exists no accepted consensus on oversight of surgical innovation, most authors support more detailed and rigorous analysis and regulation of surgical procedures that deviate further away from accepted practice. For instance, innovation involving minor modifications of a standard procedure requires either no or minimal oversight by colleagues or a surgeon-in-chief, while radically new surgical procedures should only be attempted after IRB approval or peer review with proof of concept. ${ }^{46,47}$ Second, informed consent for patients undergoing innovative surgical procedures should include descriptions of the novelty of the procedure, the surgeon's learning curve and expertise, potential outcomes of the procedure, evidence - or lack thereof - for the concept, and surgical alternatives. ${ }^{48}$ Third, most surgeons agree that attempts at an innovative procedure should be preceded by hands-on training (e.g., in cadavers) in the presence of a mentor or accreditation committee to address concerns surrounding the learning curve. Finally, innovative procedures in vulnerable populations, such as children and those who are unconscious, may require waiver of consent from institutional IRB or additional assent from the children themselves. ${ }^{49}$

A prime example of surgical innovation in contemporary head and neck surgery is transoral robotic surgery (TORS). Following evidence of efficacy and safety of robotic surgery in thoracoabdominal procedures, TORS was first created and adapted to head and neck surgery at the University of Pennsylvania. ${ }^{50}$ Years of systematic research utilizing canine and human cadaveric models, and supervised by the Food and Drug Administration (FDA), preceded use of TORS in human subjects. Prospective initial data demonstrated efficacy and safety of TORS in selected populations. ${ }^{51-53}$ Over the ensuing years, TORS has demonstrated durable disease control and acceptable complication rates in subsets of patients with squamous cell carcinoma of the oropharynx. ${ }^{54,55}$ These data culminated in FDA approval of TORS for the surgical treatment of all T1 and T2 cancers of the oral cavity, pharynx, and larynx, and all benign head and neck diseases. ${ }^{56}$

Data on utility of TORS in head and neck surgery are limited to retrospective case-control studies and prospective case series lacking participant randomization, highlighting how regulatory bodies and the clinical community adopt new technology, and the threshold for approval thereof. Some consider TORS a disruptive innovation, citing concerns regarding soaring health care costs and the extensive yet necessary investment in surgeon and ancillary staff training required of robotic instruments. ${ }^{57}$ Revisiting the ethical discussion on physician-industry relationships, the same authors also express condemnation for the aggressive and, at times, unrealistic marketing campaigns of robotic manufacturers. ${ }^{57}$ While TORS is now established as a clinical tool and is being integrated into prospective multi-institutional trials, its application and establishment within the field is instructive and complex. Of course, how this new technology continues to be adapted, refined, and utilized will remain an ever-moving target, but one informed by the basic tenets of research ethics in order to preserve the dignity and safety of the involved subjects.

\section{INFORMED CONSENT REVISITED}

While informed consent for routine clinical care and clinical research studies both intend to ensure informed, autonomous decision-making and patient/participant safety and seek to present potential risks, they differ in several important ways. Informed consent for clinical research is extensive, time-consuming, unique with 
specific details pertinent to the particular study, and scrutinized by IRBs. ${ }^{19}$ When conducting clinical research with human subjects, there are basic elements that must be included in informed consent procedures, some of which are included below:

- An explanation of the purpose and duration of research, a description of any and all involved procedures, clear identification of any experimental procedures to be implemented;

- A description of potential risks and benefits to the patient;

- Disclosure of alternative procedures or courses of treatment and an assurance of confidentiality;

- A statement that participation is completely voluntary and that refusal to participate will not be met with penalty or loss of entitled benefit;

- Circumstances in which the research project would be terminated by the investigator. ${ }^{58}$

Despite these protections, timeless problems with informed consent procedures include failure of patients and/or research participants to comprehend what they are consenting to and poor recall of study purpose and details of participation at later dates. ${ }^{59}$

A particular concern in oncologic research is the therapeutic misconception, "when individuals do not understand that the defining purpose of clinical research is to produce generalizable knowledge, regardless of whether the subjects enrolled in the trial may potentially benefit from the intervention under study." ${ }^{\prime \prime 0}$ Patients with advanced cancer in particular demonstrate an overwhelming propensity to misconstrue unproven interventions as potentially curative rather than experimental. ${ }^{61}$ The etiology of the therapeutic misconception in cancer patients is multifactorial and may be due in part to physician miscommunication, unique dynamics of the physician-patient relationship, or even specific patient-turned-participant demographics. ${ }^{61}$ Regardless, when the therapeutic misconception pervades a participant's approach to whether or not they consent to the protocol, it is probable the standard for genuine ongoing informed consent is not being met. ${ }^{62}$

The emergence of precision head and neck oncology trials has created a novel dilemma impacting clinical research informed consent. A rapidly expanding and promising field, precision medicine is already being explored in patients with recurrent or metastatic cancer in the conventional therapy for advance cancer (SHIVA) ${ }^{63}$ and molecular analysis for therapy choice $(\mathrm{MATCH})^{64}$ trials, yet formal guidelines for implementation and conversations on implications for informed consent lag. Such patients may similarly misunderstand the experimental vs therapeutic benefit of such clinical trials. ${ }^{61}$ These patients, who epitomize a vulnerable patient population, may participate in precision medicine trials expecting a cure when, in reality, they may not even be eligible to receive a targeted agent. Given these realities, clinicians and researchers need to acknowledge the unpredictability of outcomes and toxicities in precision medicine trials, to reflect on how best to emphasize these facts in an informed consent procedure, and to remain cognizant of the therapeutic misconceptions in their patients with recurrent or metastatic cancer. ${ }^{65}$

\section{CONCLUSION}

The rapidly evolving landscape of clinical research in head and neck cancer promises scientific discovery that will benefit patients and stand to improve the human condition for years to come. However, it is imperative that the head and neck oncology research community remains cognizant of the ethical conundrums posed by novel clinical investigation and respects and honors the primacy of the patients and participants upon whom such discoveries depend.

\section{REFERENCES}

1. Moss AJ, Francis CW, Ryan D. Collaborative clinical trials. N Engl J Med 2011 Mar;364(9):789-791.

2. Glickman SW, McHutchison JG, Peterson ED, Cairns CB, Harrington RA, Califf RM, Schulman KA. Ethical and scientific implications of the globalization of clinical research. N Engl J Med 2009 Feb;360(8):816-823.

3. Munchel S, Hoang Y, Zhao Y, Cottrell J, Klotzle B, Godwin AK, Koestler D, Beyerlein P, Fan JB, Bibikova M, et al. Targeted or whole genome sequencing of formalin fixed tissue samples: potential applications in cancer genomics. Oncotarget 2015 Sep;6(28):25943-25961.

4. Angus DC. Fusing randomized trials with big data. The key to self-learning health care systems? JAMA2015Aug;314(8):767-768.

5. Grumbach K, Lucey CR, Johnston SC. Transforming from centers of learning to learning health systems: the challenge for academic health centers. JAMA 2014 Mar;311(11):1109-1110.

6. Ferris RL, Blumenschein G Jr, Fayette J, Guigay J, Colevas D, Licitra L, Harrington K, Kasper S, Vokes EE, Even C, et al. Nivolumab for recurrent squamous-cell carcinoma of the head and neck. N Engl J Med 2016;375(19):1856-1867.

7. Mehanna H, Wong W, McConkey CC, Rahman JK, Robinson M, Hartley AGJ, Nutting C, Powell N, Al-Booz H, Robinson M, et al. PET-CT surveillance versus neck dissection in advanced head and neck cancer. N Engl J Med 2016;374:1444-1454.

8. D'Cruz AK, Vaish R, Kapre N, Dandekar M, Gupta S, Hawaldar R,AgarwalJ,Pantvaidya G,ChaukarD, DeshmukhA, et al. Elective versus therapeutic neck dissection in nodenegative oral cancer. N Engl J Med 2015 Nov;373(6):521-529.

9. Michmerhuizen NL, Leonard E, Kulkarni A, Brenner JC. Differential compensation mechanisms define resistance to PI3K inhibitors in PICK3CA amplified HNSCC. Otorhinolaryngol Head Neck Surg 2016;1(2):44-50.

10. Ludwig ML, Birkeland AC, Hoesli R, Swiecicki P, Spector ME, Brener JC. Changing the paradigm: the potential for targeted therapy in laryngeal squamous cell carcinoma. Cancer Biol Med 2016 Mar;13(1):87-100.

11. Birkeland AC, Yanik M, Tillman BN, Scott MV, Foltin SK, Mann JE, Michmerhuizen NL, Ludwig ML, Sandelski MM, 
Komarck CM, et al. Identification of targetable ERBB2 aberrations in head and neck squamous cell carcinoma. JAMA Otolaryngol Head Neck Surg 2016 Jun;142(6):559-567.

12. Emanuel EJ, Wendler D, Grady C. What makes clinical research ethical? JAMA 2000;283(20):2701-2711.

13. Curran WJ. The Tuskegee syphilis study. N Engl J Med 1973;289:730-731.

14. Presidential Commission for the Study of Bioethical Issues. Ethically impossible: STD research in Guatemala from 1946 to 1948, 2011. Available from: http:/ / www.bioethics.gov/cms / node/306.

15. Katz J. The Nuremberg Code. JAMA 1996 Nov;276(20):1691.

16. World Medical Association. Declaration of Helsinki. JAMA 2013 Nov;310(20):2191-2194.

17. International Ethical Guidelines for Health-Related Research Involving Humans, 2016. Available from: http:/ / cioms.ch/ ethical-guidelines-2016/WEB-CIOMS-EthicalGuidelines.pdf.

18. The Belmont Report, 1979. Available from: https://www.hhs. gov/ohrp/regulations-and-policy/belmont-report/\#xapp.

19. Federal Policy for the Protection of Human Subjects, 2017. Available from: https:/ / www.gpo.gov/fdsys/pkg/FR-201701-19/pdf/2017-01058.pdf.

20. Guidance for Institutional Review Boards and Clinical Investigators, 2016. Available from: https://www.fda.gov/ RegulatoryInformation/Guidances/ucm126420.htm.

21. Temple R, Ellenberg SS. Placebo-controlled trials and activecontrol trials in the evaluation of new treatments. Part 1: Ethical and scientific issues. Ann Intern Med 2000 Sep;133(6):455-463.

22. Ellenberg SS, Temple R. Placebo-controlled trials and activecontrol trials in the evaluation of new treatments. Part 2: Practical issues and specific cases. Ann Intern Med 2000 Sep;133(6):464-470.

23. Aspinall RL, Goodman NW. Denial of effective treatment and poor quality of clinical information in placebo controlled trials of ondansetron for postoperative nausea and vomiting: a review of published trials. BMJ 1995 Sep;311(7009):844-846.

24. Freedman B. Equipoise and the ethics of clinical research. N Engl J Med 1987 Jul;317(3):141-145.

25. Davies L, Welch HG. Increasing incidence of thyroid cancer in the United States, 1973-2002. JAMA 2006;295(18):2164-2167.

26. Leenhardt L, Grosclaude P, Cherie-Challine L, Thyroid Cancer Committee. Increased incidence of thyroid carcinoma in France: a true epidemic or thyroid nodule management effects? Report from the French Thyroid Committee. Thyroid 2004 Dec;14(12):1056-1060.

27. Mazzaferri EL. Management of low-risk differentiated thyroid cancer. Endocr Pract 2007 Sep;13(5):498-512.

28. Ito K, Miyauchi A, Kihara M, Higashiyama T, Kobayashi K, Miya A. Patient age is significantly related to the progression of papillary microcarcinoma of the thyroid under observation. Thyroid 2014 Jan;24(1):27-34.

29. Sugitani I, Toda K, Yamada K, Yamamoto N, Ikenaga M, Fujimoto $Y$. Three distinctly different kinds of papillary thyroid microcarcinoma should be recognized: our treatment strategies and outcomes. World J Surg 2010 Jun;34(6):1222-1231.

30. Xu J, Neale AN, Dailey RK, Eggly S, Schwartz KL. Patient perspective on watchful waiting/active surveillance for localized prostate cancer. J Am Board Fam Med 2012 Nov-Dec;25(6): 736-770.

31. Stack BC Jr, Angelos P. The ethics of disclosure and counseling of patient with thyroid cancer. JAMA Otolaryngol Head Neck Surg 2015 Nov;141(11):957-958.
32. Ehrhardt S, Appel LJ, Meinert CL. Trends in National Institutes of Health funding for clinical trials registered in clinical-trials. gov. JAMA 2015 Dec;314(23):2566-2567.

33. Zhang J, Yuan Y. Industry-funded clinical trials: beneficial or harmful? Clin Res Regul Aff 2015;32(4):109-112.

34. Devaiah A, Murchison C. Characteristics of NIH- and industry-sponsored head and neck cancer trials. Laryngoscope 2016 Sep;126(9): 300-303.

35. Prasad V, Berger VW. Hard-wired bias: how even doubleblinded, randomized controlled trials can be skewed from the start. Mayo Clin Proc 2015 Sep;90(9):1171-1175.

36. De Vries R, Elliott C. Why disclosure? J Gen Intern Med 2006 Jul;21(7):1003-1004.

37. Rothman DJ, McDonald WJ, Berkowitz CD, Chimonas SC, DeAngelis CD, Hale RW, Nissen SE, Osborn JE, Scully JH Jr, Thomson GE, et al. Professional medical associations and their relationship with industry: a proposal for controlling conflict of interest. JAMA 2009 Apr;301(13):1367-1372.

38. Rosenfeld RM, Shiffman RN. Clinical practice guideline development manual: a quality-driven approach for translating evidence into action. Otolaryngol Head Neck Surg 2009 Jun;140 (Suppl 1):S1-S43.

39. Sun GH. Conflicts of interest reporting in otolaryngology clinical practice guidelines. Otolaryngol Head Neck Surg 2013 Aug;149(2):187-191.

40. Norris SL, Holmer HK, Ogden LA, Burda BU. Conflict of interest in clinical practice guideline development: a systematic review. PLoS One 2011;6:e25153.

41. American Academy of Otolaryngology - Head and Neck Surgery. Clinical Practice Guidelines, 2017. Available from: http://www.entnet.org/content/clinical-practice-guidelines.

42. Unger CA, Barber MD. Studying surgical innovations: challenges of the randomized controlled trial. J Minim Invasive Gynecol 2015 May-Jun;22(4):573-582.

43. Ceelen WP. Clinical research in surgery: threats and opportunities. Eur Surg Res 2014;53(1-4):95-107.

44. Lee WT, Rocke D, Holsinger C. Surgical innovation, industry partnership, and the enemy within. Head Neck 2014 Apr;36(4):461-465.

45. Broekman ML, Carriere ME, Bredenoord AL. Surgical innovation: the ethical agenda. Medicine 2016 Jun;95(25):e3790.

46. Lieberman I, Herndon J, Hahn J, Fins JJ, Rezai A. Surgical innovation and ethical dilemmas: a panel discussion. Cleve Clin J Med 2008 Nov;75(Suppl 6):S13-S21.

47. Bernstein M, Bampoe J. Surgical innovation or surgical evolution: an ethical and practical guide to handling novel neurosurgical procedures. J Neurosurg 2004 Jan;100(1):2-7.

48. Lieberman JR, Wenger N. New technology and the orthopaedic surgeon: are you protecting your patients? Clin Orthop Relat Res 2004 Dec;429:338-341.

49. Pretz JL, Magnus D, Spain DA. Emergency innovation: implications for the trauma surgeon. J Trauma 2009 Dec;67(6):1443-1447.

50. University of Pennsylvania, Department of Otorhinolaryngology - Head and Neck Surgery. TransOral Robotic Surgery (TORS), 2017. Available from: http:/ / www.uphs.upenn.edu/ pennorl/research/tors/.

51. Weinstein GS, O'Malley BW Jr, Hockstein NG. Transoral robotic surgery: supraglottic laryngectomy in a canine model. Laryngoscope 2005 Jul;115(7):1315-1319.

52. Hockstein NG, Nolan JP, O’Malley BW Jr, Woo YJ. Robot-assisted pharyngeal and laryngeal microsurgery: 
results of robotic cadaver dissections. Laryngoscope 2005 Jun;115(6):1003-1008.

53. Hockstein NG, O'Malley BW Jr, Weinstein GS. Assessment of intraoperative safety in transoral robotic surgery. Laryngoscope 2006 Feb;116(2):165-168.

54. Grant DG, Hinni ML, Salassa JR. Oropharyneal cancer: a case for single modality treatment with transoral laser microsurgery. Arch Otolaryngol Head Neck Surg 2009 Dec;135(12): 1225-1230.

55. Dean NR, Rosenthal EL, Carroll WR, Kostrzewa JP, Jones VL, Desmond RA. Robotic-assisted surgery for primary or recurrent oropharyngeal carcinoma. Arch Otolaryngol Head Neck Surg 2010;136(4):380-384.

56. United States Food and Drug Administration. Discussion paper, Robotically-Assisted Surgical Devices, 2015. Available from: https://www.fda.gov/downloads/MedicalDevices/ NewsEvents/WorkshopsConferences/UCM454811.pdf.

57. Couch ME, Zanation A. Transoral robotic surgery: disruptive or sustaining innovation? Arch Surg 2010 Sep;145(9):907-908.

58. American Academy of Otolaryngology - Head and Neck Surgery. Elements of informed consent, 2017. Available from: http:/ / www.entnet.org/content/elements-informed-consent.

59. Nijhawan LP, Janodia MD, Muddukrishna BS, Bhat KM, Bairy KL, Udupa N, Musmade PB. Informed consent: issues and challenges. J Adv Pharm Technol Res 2013 Jul-Sep;4(3):134-140.
60. Henderson GE, Churchill LR, Davis AM, Easter MM, Grady C, Joffe S, Kass N, King NM, Lidz CW, Miller FG, et al. Clinical trials and medical care: defining the therapeutic misconception. PLoS Med 2007 Nov;4(11):e324.

61. Weeks JC, Catalano PJ, Cronin A, Finkelman MD, Mack JW, Keating NL. Patients' expectations about effects of chemotherapy for advanced cancer. N Engl J Med 2012 Oct;367(17): 1616-1625.

62. Lidz CW, Appelbaum PS, Meisel A. Two models of implementing informed consent. Arch Intern Med 1988 Jun;148(6):1385-1389.

63. LeTourneau C, Delord JP, Goncalves A, Gavoille C, Dubot C, Isambert N, Campone M, Tredan O, Massiani M, Mauborgne C, et al. Molecularly targeted therapy based on tumour molecular profiling versus conventional therapy for advanced cancer (SHIVA): a multicentre, open-label, proof-of-concept, randomised, controlled phase 2 trial. The Lancet Oncology 2015;16(13):1324-1334.

64. NCI-MATCH: Targeted therapy directed by genetic testing in treating patients with advanced refractory solid tumors, lymphomas, or multiple myeloma, 2016. Available from: https://clinical-trials.gov/show/NCT02465060.

65. Marchiano EJ, Birkeland AC, Swiecicki P, Spector-Bagdady K, Shuman A. Revisiting the therapeutic misconception in an era of precision oncology. Under Review. 